\title{
Diagnóstico do Anismus Através dos Exames de Fisiologia Anal
}

\author{
Diagnosis of the Anismus Through the Anorectal Physiology Tests
}

\author{
MARIA AUXILIADORA PROLUNGATTI CESAR ${ }^{1}$; WILMAR ARTUR KLUG ${ }^{2}$; JORGE ALBERTO ORTIZ³; \\ FANG CHIA BIN ${ }^{4}$; PERETZ KAPELHUCHNIK ${ }^{5}$
}

\begin{abstract}
1. Mestre e Doutora em Cirurgia - Área de Coloproctologia da Faculdade de Ciências Médicas da Santa Casa de São Paulo. Professor Assistente Doutor da Universidade de Taubaté; ${ }^{2}$. Professor Titular Livre Docente da Faculdade de Ciências Médicas da Santa Casa de São Paulo. Chefe da Área de Coloproctologia da Santa Casa de São Paulo;

3. Mestre em Cirurgia - Area de Coloproctologia - Pela Faculdade de Ciências Médicas da Santa Casa de São Paulo. Responsável pelo Ambulatório de Fisiologia Anal do Departamento de Cirurgia - Área de Coloproctologia da Faculdade de Ciências Médicas da Santa Casa de São Paulo; ${ }^{4}$. Mestre e Doutor em Cirurgia da Faculdade de Ciências Médicas da Santa Casa de São Paulo; ${ }^{5}$ Professor Titular Livre Docente da Faculdade de Ciências Médicas da Santa Casa de São Paulo.
\end{abstract}

\begin{abstract}
CESAR MAP; KLUG WA; ORTIZ JA; BIN FC; KAPELHUCHNIK P. Diagnóstico do Anismus Através dos Exames de Fisiologia Anal. Rev bras Coloproct, 2009;29(2): 192-196.

RESUMO: Dentre as causas de defecação obstruída, a disfunção do pubo-retal ou anismus é a mais frequente e o seu diagnóstico é feito com maior frequência através de eletromiografia , considerado como o melhor exame para o diagnóstico desta afecção. $O$ objetivo deste trabalho é verificar a influência do anismus ou contração paradoxal do pubo-retal nos exames de fisiologia anal. Para este trabalho foram estudados 40 pacientes com diagnóstico de defecação obstruída e separados em dois grupos:com e sem anismus. Todos os pacientes foram submetidos aos exames de manometria, sensibilidade retal, eletromiografia, latência do nervo pudendo e proctografia com a finalidade de observar a influência da presença de anismus nos resultados de exames de fisiologia anal. Não observamos diferenças entre os dois grupos em relação aos exames realizados, exceto na eletromiografia que é considerada padrão ouro e no ângulo ano retal na proctografia em que nos pacientes com anismus foi significantemente menor. Conclusão: a presença de anismus interferiu nos resultados dos exames de fisiologia anal apenas na eletromiografia, considerado padrão ouro para esse diagnóstico.
\end{abstract}

Descritores: Constipação; Técnicas de Diagnóstico e Procedimentos; Diagnóstico diferencial; Eletromiografia.

\section{INTRODUÇÃO}

A constipação intestinal pode ser definida como menos que três evacuações por semana ou evacuação com dificuldade, incompleta ou prolongada $(1 ; 2 ; 3)$. Pode ser classificada em dois tipos: transito colônico lento ou inércia colônica e defecação obstruída $(4 ; 5 ; 6 ; 7)$.

A defecação obstruída é a constipação que ocorre por distúrbios da dinâmica da evacuação, como inabilidade para evacuar o volume do reto, sensação de reto repleto de fezes, dor retal, descida do diafragma pélvico e esforço evacuatório (5).

Dentre as causas de defecação obstruída, a disfunção do pubo-retal ou anismus é frequente (3) e o seu diagnóstico é feito através de eletromiografia, considerado como o melhor exame para o diagnóstico desta afecção (2).

O objetivo deste trabalho é verificar a utilização dos exames de fisiologia anal no diagnóstico da contração paradoxal do pubo-retal em pacientes com constipação . 


\section{CASUÍSTICA E MÉTODO}

O trabalho realizado consistiu no estudo de 40 fichas de pacientes com constipação intestinal provenientes do ambulatório de Fisiologia anal da área de Coloproctologia da Santa Casa de São Paulo e que realizaram os exames completos de fisiologia anal . Esses pacientes foram separados em dois grupos : portadores e não portadores de anismus que foi considerado presente nas seguintes situações : aumento da atividade mioelétrica na eletromiografia; não abertura do canal anal no esforço evacuatório na proctografia e aumento da pressão de evacuação em relação à pressão de repouso na manometria anal.

Os seguintes exames foram realizados em todos os pacientes:

\section{Manometria Ano-Retal}

Utilizado o aparelho Proctosystem PL-3000 com manômetro de balão. Os exames foram realizados sem preparo intestinal, colocando-se o paciente em decúbito lateral esquerdo.

Foram consideradas a maior pressão de repouso, a maior pressão de contração e a menor pressão de evacuação no canal anal. No reto foi considerada a máxima pressão retal de repouso e a máxima pressão retal de evacuação.

\section{Sensibilidade Retal}

Avaliada através da utilização de balão confeccionado com preservativo, de aproximadamente seis centímetros de comprimento conectado à uma sonda retal e esta à uma seringa de $50 \mathrm{ml}$. Foram medidas:

a) Menor volume sensível: o primeiro volume que provocava o desejo de evacuar, que desaparecia em até 30 segundos.

b) Volume de Sensação Constante: volume que promovia um desejo de evacuar, que era permanente.

c) Volume máximo tolerável: era o volume que promovia um desejo incontrolável de evacuar.

d) Evacuação do balão: o paciente em decúbito lateral esquerdo era solicitado a realizar o esforço evacuatório para evacuar o balão. Não era ajudado inicialmente pelo examinador.

\section{Proctografia}

Realizado após a introdução de $120 \mathrm{ml}$ de contraste baritado na ampola retal com o paciente em decúbito lateral esquerdo se obtendo três radiografias na posição lateral em repouso, contração e evacuação.

$\mathrm{Na}$ avaliação das proctografias foram traçadas as seguintes linhas nas três radiografias:

a) Linha pubo-coccígea: linha traçada entre a parte superior da pube e o cóccix;

b) Canal anal: linha que se inicia no orifício anal e se estende até a junção ano-retal;

c) Eixo retal: linha marcada no eixo longitudinal do reto em sua parede posterior.

Após traçadas as linhas foram realizadas as seguintes medidas:

a) Posição de diafragma pélvico: medida entre a extremidade superior do canal anal e a linha pubo-coccígea através de uma linha perpendicular;

b) Posição perineal: medida entre a extremidade inferior do canal anal e a linha pubo-coccígea através de uma linha perpendicular;

c) Medida do canal anal;

d) Ângulo ano-retal: medido entre o eixo do reto e do canal anal no ponto de interseção das linhas.

Eletromiografia

A eletromiografia foi realizada se utilizando eletrodo de agulha concêntrica pouco menor que $0,1 \mathrm{~mm}$ de diâmetro que é introduzida no esfíncter externo do anus e através desta é medida a atividade mioelétrica no repouso, contração e esforço evacuatório

\section{Período de Latência do Nervo Pudendo}

Realizado através da introdução de luva apropriada com eletrodo na ponta do dedo indicador e em sua base. Eram dados estímulos ao nível da espinha isquiática nas proximidades do nervo pudendo, aferindo-se o tempo entre o estímulo no nervo pudendo e a resposta ao nível do ânus. Os valores considerados normais estavam no intervalo de 1,8 a $2,3 \mathrm{~ms}$.

A análise estatística se fez através do teste $\mathrm{t}$ de Student para comparação de médias, controlado pelo teste de Levene para igualdade das variâncias.

\section{RESULTADOS}

O diagnóstico do anismus ocorreu em 19 dos 40 pacientes estudados.

A eletromiografia diagnosticou o anismus em todos os 19 pacientes com diagnóstico final de anismus. A mesma alteração foi vista através da proctografia 
em oito dos 19 pacientes $(42,10 \%)$. Não existiram pacientes diagnosticados pela proctografia ou através da manometria que não estivessem alterados pela eletromiografia.

Os pacientes considerados portadores de anismus foram comparados aos demais pacientes constipados.

Não observamos diferenças estatisticamente significantes nos pacientes diagnosticados como portadores de anismus em relação aos outros pacientes constipados não portadores de anismus quando observamos a manometria, sensibilidade retal e latência do nervo pudendo (Tabelas 1a 3).

Observando as proctografias de portadores e não portadores de anismus, a única diferença estatisticamente significante foi em relação ao ângulo anoretal na contração (Tabela 4).

\section{DISCUSSÃO}

A existência do anismus como mecanismo de defecação obstruída na constipação intestinal é controverso (6), alguns autores consideram o aumento da atividade mioelétrica mais um achado (8). Contra os autores que sugerem a ausência de anismus está o fato de que pacientes com anismus tem melhora da dificuldade evacuatória quando se tratam através do biofeedback e algo que não existe não melhoraria com nenhum tratamento. Outro fator a favor da existência do anismus é que este diagnóstico esteve presente em vários pacientes em diferentes momentos e em diferentes exames .

Nesta casuística a contração paradoxal do pubo-retal foi diagnosticada em 19 pacientes $(47,5 \%)$ e estes foram avaliados através dos métodos de fisio-

Tabela 1 - Médias de pressões anais e retais em constipados portadores ou não de anismus.

\begin{tabular}{lccc}
\hline Manometria ano-retal & Presença de anismus & Ausência de anismus & p \\
\hline Pressão máxima de repouso & $62,79 \pm 18,01$ & $60,24 \pm 19,14$ & $\mathrm{Ns}$ \\
Pressão máxima de contração & $127,10 \pm 49,33$ & $137,90 \pm 56,54$ & $\mathrm{Ns}$ \\
Pressão mínima de evacuação & $37,58 \pm 11,83$ & $30,81 \pm 12,3$ & $\mathrm{Ns}$ \\
Pressão retal de repouso & $13,16 \pm 8,96$ & $13,52 \pm 15,32$ & $\mathrm{Ns}$ \\
Pressão retal de evacuação & $56,63 \pm 21,06$ & $56,90 \pm 23,00$ & $\mathrm{Ns}$ \\
Zona de hiperpressão & $2,99 \pm 0,77$ & $3,08 \pm 0,72$ & $\mathrm{Ns}$ \\
\hline
\end{tabular}

Tabela 2 - Médias de sensibilidade retal em constipados portadores ou não de anismus.

\begin{tabular}{lccc}
\hline Sensibilidade retal & Presença de anismus & Ausência de anismus & p \\
\hline $\begin{array}{l}\text { Volume que provoca o } \\
\text { primeiro desejo de evacuar }\end{array}$ & $65,53 \pm 28,91$ & $66,76 \pm 28,20$ & Ns \\
Volume de sensação constante & $130,79 \pm 50,34$ & $126,9 \pm 33,82$ & Ns \\
Volume máximo tolerável & $297,37 \pm 73,55$ & $311,43 \pm 147,28$ & Ns \\
\hline
\end{tabular}

Tabela 3 - Médias da latência do nervo pudendo em constipados portadores ou não de anismus.

\begin{tabular}{lccc}
\hline Latência do nervo pudendo & Presença de anismus & Ausência de anismus & p \\
\hline Direita & $1,99 \pm 0,54$ & $2,12 \pm 0,60$ & $\mathrm{Ns}$ \\
Esquerda & $2,10 \pm 0,68$ & $2,36 \pm 0,82$ & $\mathrm{Ns}$ \\
\hline
\end{tabular}


Tabela 4 - Proctografias em portadores ou não de anismus.

\begin{tabular}{lcrc}
\hline Medidas de proctografia & Presença de anismus & Ausência de anismus & p \\
\hline Ângulo ano-retal no repouso & $100,70 \pm 11,08$ & $110,00 \pm 18,29$ & $\mathrm{Ns}$ \\
Comprimento do canal anal no repouso & $3,79 \pm 1,09$ & $3,21 \pm 1,44$ & $\mathrm{Ns}$ \\
Posição do diafragma pélvico no repouso & $6,22 \pm 2,31$ & $6,25 \pm 2,28$ & $\mathrm{Ns}$ \\
Posição perineal no repouso & $8,96 \pm 2,32$ & $8,79 \pm 2,00$ & $\mathrm{Ns}$ \\
Ângulo ano-retal na contração & $79,74 \pm 10,26$ & $89,33 \pm 14,28$ & p $<0,05$ \\
Comprimento do canal anal na contração & $5,59 \pm 2,22$ & $7,16 \pm 6,07$ & $\mathrm{Ns}$ \\
Posição do diafragma pélvico na contração & $4,32 \pm 1,49$ & $3,95 \pm 1,75$ & $\mathrm{Ns}$ \\
Posição perineal na contração & $7,50 \pm 0,97$ & $7,42 \pm 1,90$ & $\mathrm{Ns}$ \\
Ângulo ano-retal na evacuação & $111,74 \pm 9,70$ & $117,67 \pm 14,06$ & $\mathrm{Ns}$ \\
Comprimento do canal anal na evacuação & $2,28 \pm 0,76$ & $2,31 \pm 1,58$ & $\mathrm{Ns}$ \\
Posição do diafragma pélvico na evacuação & $6,41 \pm 2,39$ & $7,06 \pm 2,49$ & $\mathrm{Ns}$ \\
Posição perineal na evacuação & $7,81 \pm 1,92$ & $8,80 \pm 2,17$ & $\mathrm{Ns}$ \\
\hline
\end{tabular}

logia anal e comparados com outro grupo de constipados, mas que não tinham este diagnóstico. Não existiram pacientes diagnosticados pela proctografia ou através da manometria que não estivessem alterados pela eletromiografia .

A eletromiografia foi o exame de maior valor utilizado no diagnóstico da contração paradoxal do pubo-retal, e esse diagnóstico era feito quando ocorria um aumento da atividade mioelétrica na tentativa de esforço evacuatório. O diagnóstico de contração paradoxal do pubo-retal através da proctografia ocorreu em oito dos 19 pacientes $(42,10 \%)$ que foram diagnosticados na eletromiografia, não sendo considerado por nós como melhor exame para o diagnóstico ainda mais que neste estudo não ocorreu nenhum paciente que não apresentasse contração paradoxal do pubo-retal na eletromiografia e apresentasse ou na manometria, ou na proctografia. Existem também relatos de que existiria uma associação da contração paradoxal do pubo retal com a sensação retal (9).

É preciso considerar que o paciente pode se contrair na eletromiografia devido ao incomodo do exame que foi feito através de agulha, mas devemos também considerar que a proctografia também é exame constrangedor que inibe o paciente e, no entanto, houve menor incidência de contração paradoxal do puboretal na proctografia.

\section{CONCLUSÃO}

O exame com maior sensibilidade no diagnóstico do anismus foi a eletromiografia, mas o diagnóstico tambem ocorreu através da manometria e proctografia com menor sensibilidade.

\footnotetext{
ABSTRACT: Amongst the causes of outlet obstruction, the anismus is most frequent and its diagnosis is made more frequently through electromyografy, considered as optimum examination for this diagnosis. The objective of this work is to verify the influence of anismus in the examinations of anal physiology. For this work 40 patients with diagnosis of outlet obstruction were separate in two groups had been studied: with and without anismus. All of the patients were submitted to manometric exams, rectal sensibility, electromyography, pudendal latency and defecography with the purpose of serving which ones exams would be important in the presence or not of the anismus. We didn't observe differences between the two groups in relation to the carried through examinations, except in the electromyography that is considered standard gold and in the angle retal year in the defecography where in the patients with anismus it was significantly lesser. Conclusion: the presence of anismus intervened with the results of the examinations of anal physiology only in the electromyography, considered standard gold for this diagnosis.
}

Key words: Constipation; Diagnostic Techniques and Procedures; Diagnosis, Differential; Electromyography. 


\section{REFERÊNCIAS}

1. Vaccaro, C.A.; Cheong, D.M.O.; Wexner, S.D.; Nogueras, J.J.; Salanga, V.D.; Hanson, M.R.; Phillips, R.C. - Pudendal neuropathy in evacuatory disorders. Dis. Colon. Rectum., 38:166-71, 1995.

2. Sschouten, W.R.; Briel, J.W.; Auerda, J.J.A.; Vandam, J.H.; Gosselink, M.J.; Ginai, A.Z.; Hop, W.C.J. — Anismus: fact or ficcion? Dis. Colon. Rectum., 40:1033-41, 1997.

3. Pfeifer, J.; Teoh, T-A.; Salanga, V.D.; Agachan, V.F.; Wexner, S.D. - Comparative study between intra-anal sponge and needle electrode for electromyographic evaluation of constipated patients. Dis. Colon. Rectum., 41:1153-7, 1998.

4. Bleijenberg, G.; Kuijpers, H.C. - Biofeedback treatment of constipation: a comparison of two methods. AJG, 89(7):10216, 1994.

5. Camilleri, M. - Functional gastrointestinal disorders: novel insights and treatments. Medscape Gastro. J., 1(1):1-13, 1999.

6. Mellgren, A. - Diagnosis and treatment of constipation. Eur. J. Surg., 161:623-34, 1995
7. Koutsomanis, D.; Lennard-Jones, J.E.; Roy, A.J.; Kamm, M.A. - Controlled randomised trial of visual biofeedback versus muscle training without a visual display for intractable constipation. GUT, 37:95-9, 1995.

8. Athanasiadis, G.; Weyand, G.; Kuprian, A.; Und Koller, A. Welche Rolle spielen der M. pubococcygeus und M. puborectalis bei patientin mit Obstruktiven Defakationsstorungen? Eine elektromyographische studie. Der. Chirurg., 66(10):974-81, 1995.

9. Lopez, A.; Holmstrom, B.; Nilson, B.Y.; Dolk, A.; Johanson, C.; Schultz, I.; Zetterstrom, J.; Mellgren, A. - Paradoxal sphincter reaction is influenced by rectal filling volume. Dis. Colon. Rectum., 41:1017-22, 1998.

\section{Endereço para correspondência:}

MARIA AUXILIADORA PROLUNGATTI CESAR

Rua Vinicius de Moraes , 434 - Campos do Conde I

CEP 12120000

Tremembé-SP

E-mail: prolungatti@uol.com.br 